\title{
Verification of the Precious Metals Market Effectiveness - Gold and Silver
}

\author{
Krzysztof PIASECKI and Michał Dominik STASIAK*
}

Poznan University of Economic and Business, Poznan, Poland; krzysztof.piasecki@ue.poznan.pl; michal.stasiak@ue.poznan.pl

* Correspondence: michal.stasiak@ue.poznan.pl

\begin{abstract}
The requirement for using technical analysis and for constructing algorithmic trading system based on it, is the assumption about possible dependencies between historical data and the future direction of a rate change. Making this kind of assumption leads to rejecting the hypothesis of market effectiveness. In the article a verification method for the assumption is presented for the case of precious metals - gold and silver. The base of proposed method is statistical testing performed on the binary course representation, which is more precise than the candlestick representation. The article also presents an analysis of obtained results in terms of possible construction of HFT systems.
\end{abstract}

Keywords: market effectiveness; high frequency econometric; technical analysis; investment decision support

JEL Classification: F14; G11; G14; C49

\section{Introduction}

For many years there has been a dispute over the justification for the use of technical analysis methods (Peters 1991). Due to the lack of positive rates of return generated by many traditional methods (Kirkpatrick and Dahlquist 2010) and the difficulty of statistical verification of others (e.g. those based on visual analysis, such as Elliott's wave detection (Frost and Prechter 2005), many researchers are inclined to adopt the hypothesis of market effectiveness. The hypothesis was formulated by Fama (Fama and Malkiel 1970) and states that the price of a given asset reflects the current situation on the market. All versions of the hypothesis assume the lack of effectiveness of technical analysis methods. The effective market hypothesis has been repeatedly tested for various financial instruments. However, all research was conducted based on quotations expressed in a candlestick representation and for a specific timeframe (e.g. for daily data (García, Gaytán, and Wolfskill 2012)). The use of quotations in the candlestick format leads to a poor credibility of results, especially when examining the relationship between small-scale changes that are crucial in the construction of HFT systems. For this reason, the article proposes a method of verifying the existence of statistical relationships between historical data and the current direction of exchange rate changes. The method is based on a binary representation of the course (Stasiak 2016) and statistical tests recommended by NIST (Rukhin et al. 2010). The results of the silver and gold exchange rate analysis expressed in dollars (XAU/USD and XAG/USD) were then used to verify the possibility of constructing HFT systems.

The article is organized in the following way. After a short introduction, the second chapter presents the concept of a binary representation of the course. In Section 3, a schema for verifying possible dependences between subsequent course changes is presented. Chapter 4 presents the results of statistical analysis and conclusions regarding the possibility of constructing HFT systems. In Chapter 5, the most important research results are discussed and summarized.

\section{Binary Representation}

Due to the high volatility of quotations (changes often occur even every 1, 2 seconds) and the existence of the noise phenomenon (Logue and Sweeney 1977; Lo, Mamaysky, and Wang 2000), while 
analyzing the financial data, it is necessary to use appropriate representation. The vast majority of research (including those regarding effectiveness of a given market) is carried out based on quotations of an asset expressed in a candlestick representation. In such representation, the course in a given timeframe is described by 4 parameters: the opening price, closing price and the maximum and minimum exchange rate. In fact, the intensity of changes in a certain range depends on many factors (e.g. time of day). Also, in case of events influencing the quotations of a given resource, we often register large fluctuations of the exchange rate in a short time. Therefore, the representation dependence on a set time period leads to a loss of many valuable information about the variability 'inside' the candle during high investors' activity and to registering many small-size candles (which are irrelevant to the analysis), during periods of reduced investors' activity. This phenomenon is especially important in the study of course changes with a small range, the results of which are used in HFT systems which are based on the statistical advantage of transactions that resulted in profit over those resulting in a loss. Even within 1 minute (the smallest timeframe used), there may be several changes that are not included in the candlestick analysis. However, these changes may result in making or ending many transactions and, as a consequence, the operation of a HFT system may cause losses, despite the fact that the analysis of candlestick data indicates a profit. Therefore, the use of a candlestick representation leads to imprecise and ambiguous results.

Due to the above stated disadvantages of the candlestick representation, a more accurate binary representation was used to analyze the quotation efficiency of selected resources. The concept of a binary representation is based on the assumption that, rather than time, the direction and range of changes stand as a base for the course description.

The course binarization approach was first used in the 1930s, in order to build and analyze graphs in the so-called point-symbolic method (De Villiers 1933). Unfortunately, this method of course representation was replaced by the candlestick representation. The basis of the binary representation (Fama and Malkiel 1970) is the discretization of an exchange rate, with a given discretization unit. The representation is described by a sequence $\left\{\varepsilon_{i}\right\}_{i=1}^{n}$. Figure 1 shows an example of the course binarization algorithm. This algorithm assigns ' $O$ ' to the ensuing sequence element $\varepsilon_{i}$ if the rate drops by the unit of discretization, or the value ' 1 ' in case of an analogous increase. If price gaps occur, the algorithm checks what value would have been achieved, and then analyzes the rate relative to the first price after the price gap. As a result of the algorithm's operation, the exchange rate can be presented in the form of a binary sequence. The use of a binary representation eliminates time periods characterized by a non-volatility of the course (e.g. nights), and records all changes of a certain range in the times of investors' high activity.

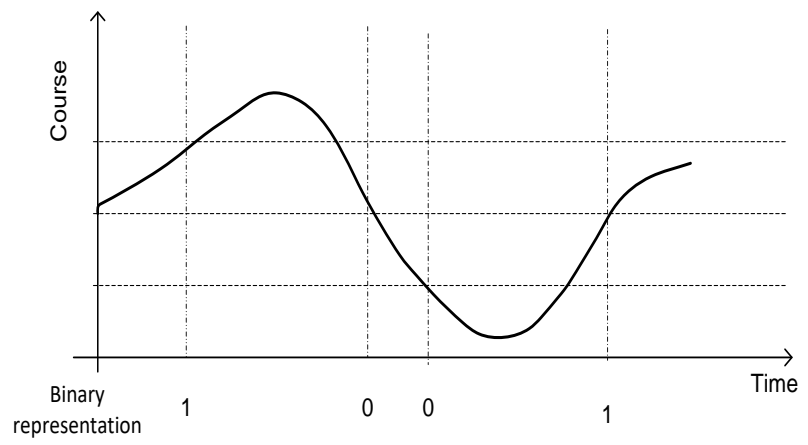

Figure 1. Course in binary representation.

\section{Research of Possible Relations Between Ensuing Rate Changes}

The character of quotation changes depends on the assumed discretization unit. Assuming too small discretization unit can lead to registering noise (random fluctuations of a small range). On the other hand, taking too big discretization unit can lead to a loss of informative value, which will make the obtained representation totally worthless. Because of above stated reasons, statistical verification of binary representations was performed, obtained for all discretization units from the range of 10 
pips (the accuracy of XAU/USD and XAG/USD quotations, with the step of 10 pips, to the value for which the obtained number of changes in the binary representation is lower than 128 (that is, the value of the sample below which it is impossible to perform all the tests).

For each binary representation (binary sequence), three statistical tests were performed, that is: Monobit Test (Chung 1979; Pitman 1993), Series Test (Gibbons 1985; Godbole and Papastavridis 1994) and The Longest Series in the Block Test (David and Burton 1962). Procedures of these tests are presented in the Appendix. Each of the tests verifies a following null hypothesis:

\section{$H_{0}$ : Observed binary representation is a sequence of unpredictable values.}

Which is opposed by the alternative hypothesis:

$H_{1}$ : Observed binary representation is a sequence of predictable values.

The verification of null hypothesis is conducted for an assumed significance level $\alpha=0.01$. If at least one of the applied tests rejects the null hypothesis, then we conclude that the binary representation is, in fact, a sequence of predictable values and can stand as a premise to predict future exchange rate trajectory changes.

\section{Study of Precious Metals Exchange Rate Changes}

In order to perform appropriate research, a dedicated software in $\mathrm{C}++$ and Mq14 languages was written. Research uses tick data of gold and silver exchange rate quotations, expressed in American dollar (XAU/USD and XAG/USD), taken from Ducascopy broker. Data consist of quotations from a six-year time period from 01.01.2013 to 01.01.2019. The time period was chosen due to the changes introduced in recent years on the market, that is the development of tele-informatic devices (e.g. MetaTrader and JForex platforms, etc.), allowing for making transactions in time close to real (the realization time is expressed in ms) and market automation support (based on MQL4 and Java languages), etc. A huge influence on the character of course changes also recently stems from the reduction of spreads and minimal contributions. Lower spreads allow for transactions of a smaller range, and lower minimal contributions opened the market for a wider group of investors. In this context, analyzing older data can introduce distortions in current regularities to be found in today's investors' behaviors.

\subsection{Research results}

As a result of analyzing the XAU/USD security, for all discretization units from range of 1 to 547 pips, we reject the hypothesis suggesting an unpredictable character of course changes for the assumed significance level. For most of the cases the Monobit Test indicates an unpredictable character of changes, yet for all discretization units from before mentioned range, remaining two tests Series Test and Test for the Longest Run of Ones in a Block Test) reject the hypothesis of lack of dependencies between historical quotations and future change direction. This kind of result suggests that the number of registered decreases and increases is comparable and does not deviate from the number of changes registered in a truly random sequence, yet the order and number of particular change schemas definitely deviates from statistics of truly random sequences. This results indicates the existence of dependencies between historical data and the current direction of a change. Obtained results therefore justify the possibility of applying advanced technical analysis methods (e.g. prediction models dedicated to a binary representation (e.g. BSM (Stasiak 2016), BFMS (Stasiak 2017)). One can also conclude that using discretization units higher than 547 pips, the loss of informative value of the binary representation is so significant, that it makes it impossible to assess the future direction of a change. This means that for transactions in which the SL and TP levels are distanced from each other by the value higher than 547 pips, the probability of achieving a profit is not possible to be assessed. 

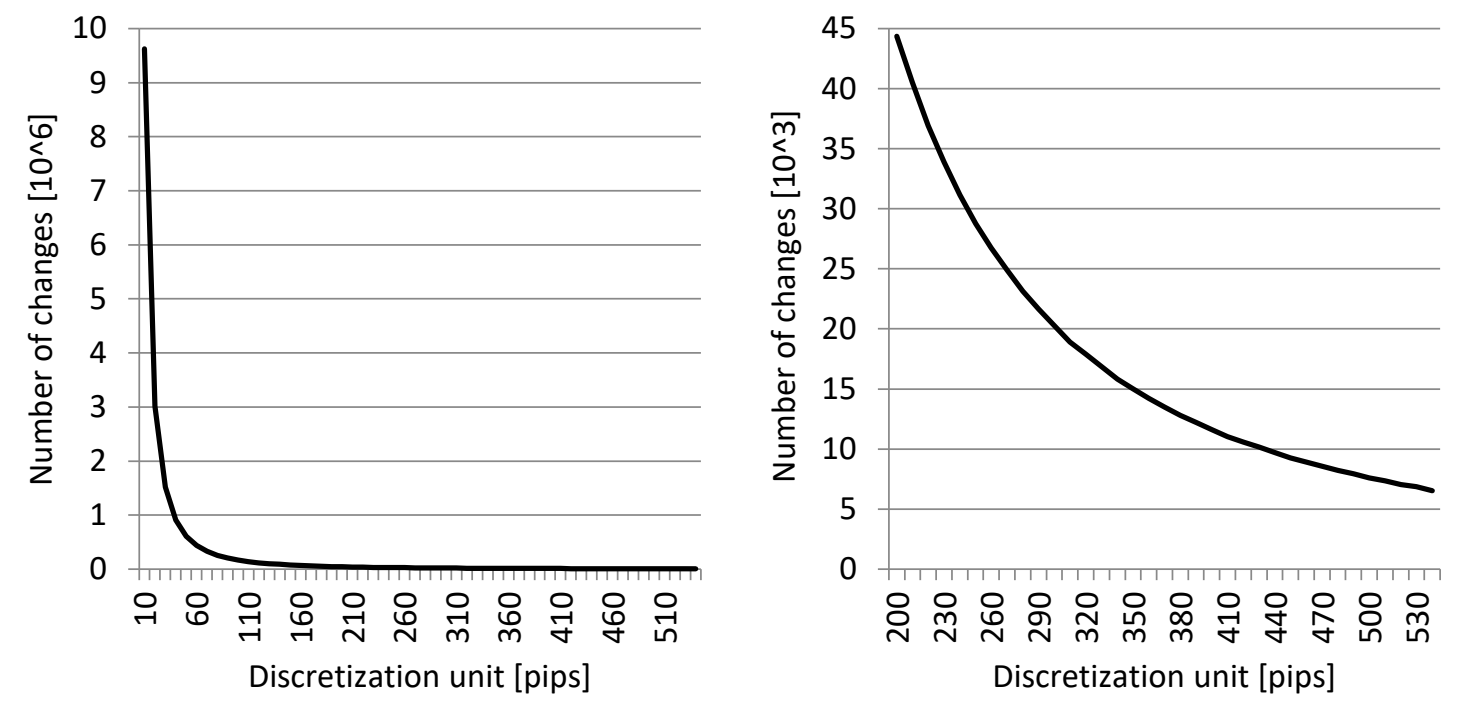

Figure 2. Number of changes (in millions) observed in the research period, depending on the discretization unit: a) for all positively verified discretization unit (1-547 pips); b) for a chosen interval discretization unit (200-547 pips).

In the Figure 2a we can see the number of observed changes in a six-year period, depending on the value of used discretization unit. This kind of analysis allows for an assessment of the number of potential transaction signals for constructed HFT system, based on a binary representation. Figure $2 \mathrm{~b}$ presents results for these discretization units, which are at least four times higher than the average spread offered by one of the most popular brokers (ICMarkets).

Analogous research was performed for the XAG/USD valor. For the silver exchange rate and for all discretization units from the range of 1 to 281 pips, the hypothesis about unpredictable character of changes was rejected at assumed level of significance. Also in this case, only the monobit test indicates an unpredictable character of rate changes. Obtained results show, that for transactions in which SL and TP are separated from each other by a higher value, from the point of view of historical data analysis we cannot assess the probability of success.

In the Figure 3 we can see the number of changes observed in a binary representation for these discretization units, for which the binary representation retains its informative value. Results allow for formulating a conclusion that the transactions in which SL and TP levels are distanced by value higher than 281 pips, from the point of view of historical data analysis we cannot assess the probability of reaching a potential profit. 

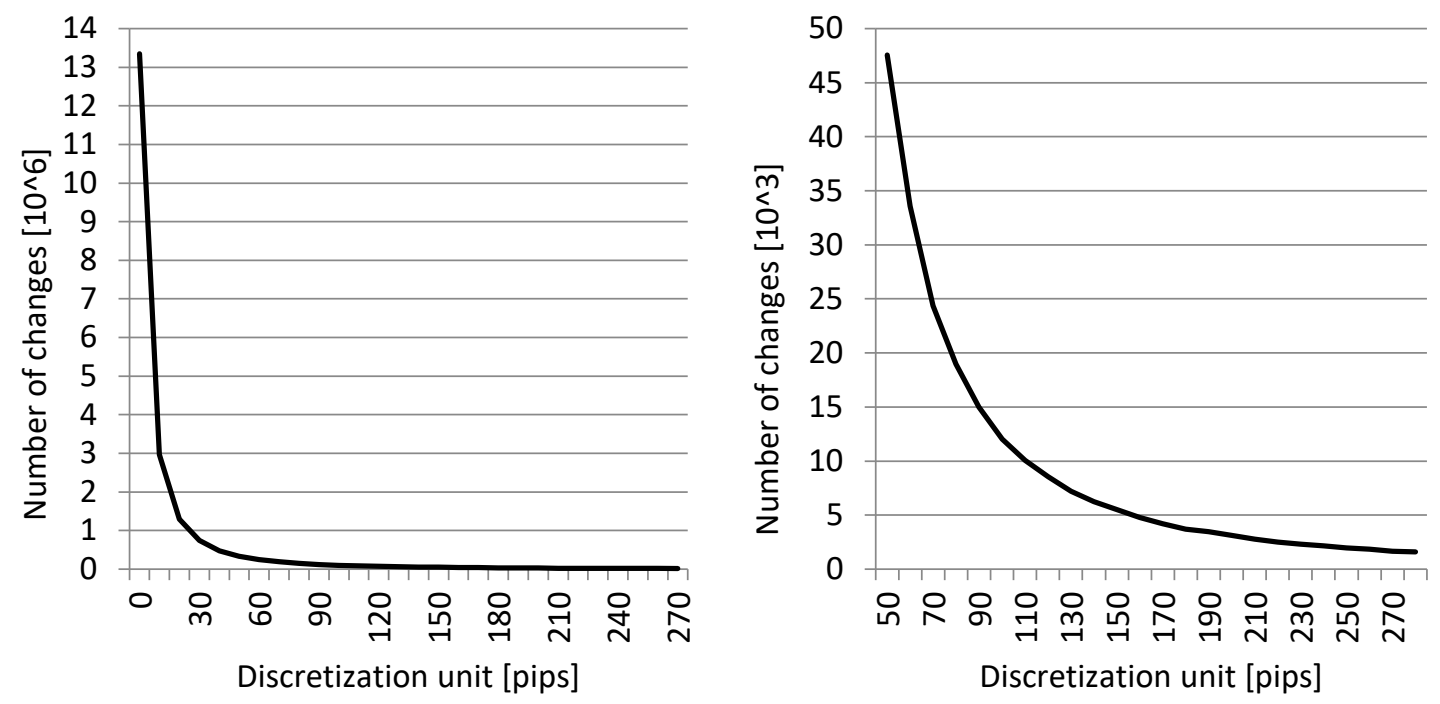

Figure 3. Number of changes (in millions) observed in the research period, depending on the discretization unit: a) for all positively verified discretization unit (1-281 pips); b) for a chosen interval discretization unit (50-281 pips).

\subsection{Practical application of obtained results in constructing HFT systems}

Binary representation also allows for a simple implementation in HFT systems (Piasecki and Stasiak 2019). In traditional technical analysis of a candlestick chart, there arise a problem of proper TP and SL levels appointment and credible probability assessment of investment resulting in actual profit. Because of this, in many publications those parameters are omitted, and only levels of future rate increase or decrease in a given time is assessed, (which makes it impossible to evaluate this kind of strategy).

In the binary representation, course change by one discretization unit can be connected with TP and SL levels. Let us consider an exemplary HFT system, in which TP and SL parameters are distanced from the opening price by one discretization unit $(\delta)$. In this situation, the value of possible loss or profit (including the spread (spr)) of a single transaction can be calculated with the following formula:

$$
\begin{gathered}
\text { Profit }=(\delta-s p r) * v, \\
\text { Loss }=(\delta+s p r) * v,
\end{gathered}
$$

where $v$ is the transaction size expressed in Lot (Lot is a unit describing the transaction size). Assuming the same value of each transaction, this parameter only scales the position value and has no influence on considerations presented in the article. Based on the formulas (1) and (2), after simple transformations, we can indicate the limit probability assessment of the future direction change $P_{g}$, above which the HFT system will generate a constant profit:

$$
P_{g}=\frac{(\delta+s p r)}{2 \delta}
$$

By the formula (3) and knowing the offered spread and chosen discretization unit, we can then describe the required level of probability assessment for future direction of a change, which has to be guaranteed by the applied technical analysis method in order for an investment to be characterized by a positive return rate. If this probability is higher than the threshold value, then the system will generate profit. 
In the presented article, authors focused on presenting a solution schema for the problem formulated in a following way: do the precious metals exchange rates (XAU/USD and XAG/USD) show any dependencies between the historical and future quotations, and for which discretization units this kind of dependencies are visible. Performed research showed that for discretization units from the range of 1-547 pips (for gold), there exist a possibility of applying technical analysis in order to predict the exchange rate trajectory. Average spread offered by one of the most popular brokers (ICMarkets) equals 20 pips for XAU/USD. For higher discretization unit, the lower the spread influence. According to the formula (3), for example for the discretization unit of 420 pips, the level of prediction equal to 0.53 of accurate predictions, allows for obtaining a positive return rate.

The authors' current research focuses on developing new prediction models dedicated to the precious metals market in order to build and analyze the risk of algorithmic trading systems for this market.

\section{Summary}

In the article, the verification method for possible dependencies between the current change direction and the historical quotations of precious metals exchange rates (XAU/USD and XAG/USD) is presented. In the researched method, statistical testing and binary rate representation was used, which eliminates the loss of informative value usually created by using the candlestick representation.

As a result of the research, the hypothesis about the market effectiveness for XAU/USD and XAG/USD was rejected and existence of possible dependencies between historical data and current change direction was proved. These conclusions justify the application of technical analysis methods like state modeling etc. in assessing the probability of the direction of future changes.

In the article, for researched securities, the range of discretization units was also indicated, for which the binary representation retains its informative value and can be used in technical analysis. Moreover, minimal requirements for prediction tools were also pointed out, by fulfilling which the investor achieves a positive return rate.

\section{Appendix}

Let the sequence of random values $\left\{\varepsilon_{i}\right\}_{i=1}^{n}$ describe the observed binary representation of given currency pair. Function $\operatorname{erf}(\cdot)$ is defined by following formula:

$$
\operatorname{erf}(x)=\frac{2}{\pi} \int_{-\infty}^{x} e^{-\frac{t}{2} \cdot t^{2}} d t
$$

Monobit Test (Chung 1979; Pitman 1993)

Statistic of this test is given by the relation:

$$
S_{o b s}=\frac{2 \cdot \sum_{i=1}^{n} \varepsilon_{i}-n}{\sqrt{n}} .
$$

If the below stated condition is met:

$$
p_{\text {value }}=2 \cdot\left(1-\operatorname{erf}\left(\left|S_{\text {obs }}\right|\right)\right)<\alpha,
$$

then we can say that there is a basis to reject the null hypothesis $H_{0}$ in favor to the alternative hypothesis $H_{1}$.

Series Test (Gibbons 1985; Godbole and Papastavridis 1994)

$$
V_{o b s}=1+\sum_{i=1}^{n-1}\left|\varepsilon_{i+1}-\varepsilon_{i}\right| .
$$


Statistic of this test is given by the relation:

$$
Z_{o b s}=\frac{V_{o b s}-E\left(V_{o b s}\right)}{\sqrt{D\left(V_{o b s}\right)}}
$$

where:

$$
\begin{gathered}
V_{o b s}=1+\sum_{i=1}^{n-1}\left|\varepsilon_{i+1}-\varepsilon_{i}\right| . \\
E\left(V_{o b s}\right)=2 \cdot \frac{n_{1} \cdot\left(n-n_{1}\right)}{n}+1, \\
D\left(V_{o b s}\right)=2 \cdot \frac{n_{1} \cdot\left(n-n_{1}\right)\left(2 \cdot n_{1} \cdot\left(n-n_{1}\right)-n\right)}{(n-1) \cdot n^{2}}, \\
n_{1}=\sum_{i=1}^{n} \varepsilon_{i} .
\end{gathered}
$$

If the following condition is met:

$$
p_{\text {value }}=2 \cdot\left(1-\operatorname{erf}\left(\left|Z_{\text {obs }}\right|\right)\right)<\alpha,
$$

then we can say that there is a basis to reject the null hypothesis $H_{0}$ in favor to the alternative hypothesis $H_{1}$.

Test for the Longest Run of Ones in a Block (David and Barton 1962).

By the term of 'series' we understand a random and possibly longest subsequence of ensuing ' 1 '. In order to perform the Longest Series Test, the binary representation $\left\{\varepsilon_{i}\right\}_{i=1}^{n}$ is divided into $N$ subsequences, consisting of $M$ ensuing elements of $\left\{\varepsilon_{i}\right\}_{i=1}^{n}$ sequence. Each representation subsequence obtained this way is called a block. Next, we appoint probabilities $\pi_{m}^{*}$ of events $\Pi_{m}^{*}$ defined this way, that the length of the longest series in $M$-element block is equal to $m=0,1,2, \ldots, M$. Probabilities $\pi_{m}^{*}$ are appoited based on following formulas:

$$
\begin{gathered}
\pi_{m}^{*}= \begin{cases}q_{0} & m=0 \\
q_{m}-q_{m-1} & m>0^{\prime}\end{cases} \\
q_{m}=\frac{1}{2^{M}} \sum_{r=0}^{M} \sum_{j=0}^{U(r)}(-1)^{j} \cdot\left(\begin{array}{c}
M-r+1 \\
j
\end{array}\right) \cdot\left(\begin{array}{c}
M-j \cdot(m+1) \\
M-r
\end{array}\right) \\
U(r)=\min \left\{M-r+1, \text { entier }\left(\frac{r}{m+1}\right)\right\},
\end{gathered}
$$

Next, we indicate such minimal values of $k, l$ parametes, that the following conditions are met:

$$
\begin{gathered}
\sum_{t=0}^{k} \pi_{t}^{*} \cdot N>5, \\
\sum_{t=M-l}^{M} \pi_{t}^{*} \cdot N>5,
\end{gathered}
$$

Next step consist of appointing a sequence of events $\left\{\Pi_{s}\right\}_{s=k}^{l}$ described by relations (Revesz 1990): 


$$
\Pi_{s}=\left\{\begin{array}{lr}
\coprod_{t=0}^{k} \Pi_{t}^{*} & s=k \\
\Pi_{s}^{*} & k<s<M-l \\
\coprod_{t=M-l}^{M} \Pi_{t}^{*} & s=M-l
\end{array},\right.
$$

Probability $\pi_{s}$ of an event occurring is calculated the following way:

$$
\pi_{s}=\left\{\begin{array}{lr}
\sum_{t=0}^{k} \pi_{t}^{*} & s=k \\
\pi_{s}^{*} & k<s<M-l . \\
\sum_{t=M-l}^{M} \pi_{t}^{*} & s=M-l
\end{array} .\right.
$$

In the last step, for each event $\Pi_{s} s=k, k+1, \ldots, M-l$ we describe the number of blocks $v_{s}$, corresponding to given event. Statistic of the thest of Longest Series in the Block is defined in the following way:

$$
V_{o b s}=\sum_{s=k}^{M-l} \frac{\left(v_{s}-N \pi_{s}\right)^{2}}{N \pi_{s}}
$$

If the following condition is met:

$$
p_{\text {value }}=1-\chi^{2}\left(V_{\text {obs }}, M-k-l+1\right)<\alpha,
$$

where:

$$
\chi^{2}(x, N)=\frac{\int_{0}^{x} t^{N / 2-1} e^{-t} d t}{2^{N / 2-1} \cdot \int_{0}^{\infty} t^{N / 2-1} e^{-t} d t}
$$

then we can say that there is a basis to reject the null hypothesis $H_{0}$ in favor to the alternative hypothesis $H_{1}$.

\section{References}

Chung Kai L. 1979. Elementary Probability Theory with Stochastic Processes. New York: Springer.

David N., and Barton D. E. 1962. Combinatorial Chance. London: C. Griffin.

De Villiers Victor. 1933. The Point and Figure Method of Anticipating Stock Price Movements: Complete Theory and Practice. Windsor books.

Fama Eugene F., and Malkiel Burton G. 1970. Efficient Capital Markets: A Review of Theory and Empirical Work. The Journal of Finance: 43, 383-417. https://doi.org/10.1111/j.1540-6261.1970.tb00518.x.

Frost Alfred J., and Prechter Robert R. 2005. Elliott wave principle: key to market behavior. Elliott Wave International. García Bernardo Q., Gaytán Jesus C., and Wolfskill Lawrence A. 2012. The Role Of Technical Analysis In The Foreign Exchange Market. Global Journal of Business Research: 6, 17-22.

Gibbons Jean D. 1985. Nonparametric Statistical Inference. Marcel Dekker.

Godbole Anant P., Papastavridis Stavros G. (eds) 1994. Runs and patterns in probability: Selected papers. London: Kluwer.

Kirkpatrick Charles D., and Dahlquist Julie A. 2010. Technical analysis: the complete resource for financial market technicians. FT press.

Lo Andrew W., Mamaysky Harry, and Wang Jiang. 2000. Foundations of technical analysis: Computational algorithms, statistical inference, and empirical implementation. The Journal of Finance: 55, 1705-1770. https://doi.org/10.3386/w7613.

Logue Dennis E., and Sweeney Richard J. 1977. White noise in imperfect markets: the case of the franc/dollar exchange rates. The Journal of Finance: 32, 761-768. https://doi.org/10.1111/j.1540-6261.1977.tb01986.x. 
Peters Edgar E. 1991. Chaos and Order in the Capital markets: A New View of Cycles, Prices, and Market Volatility. New York: Wiley.

Piasecki Krzysztof, and Stasiak Michal D. 2019. The Forex Trading System for Speculation with Constant Magnitude of Unit Return. Mathematics: 7(7). https://doi.org/10.3390/math7070623.

Pitman Jim. 1993. Probability. New York: Springer-Verlag.

Rukhin A. 1. et al. 2010. Statistical test suite for random and pseudorandom number generators for cryptographic applications. Gaithersburg: NIST.

Stasiak Michal D. 2016. Modelling of currency exchange rates using a binary representation. In Advances in Intelligent Systems and Computing. Cham: Springer International Publishing, Vol. 524, pp. 153-161. https://doi.org/10.1007/978-3-319-46592-0_13.

Stasiak Michal D. 2017. Modelling of Currency Exchange Rates Using a Binary-Wave Representation. In Advances in Intelligent Systems and Computing. Cham: Springer International Publishing, Vol. 657, pp. 27-37. https://doi.org/10.1007/978-3-319-67223-6_3. 